\title{
ON THE EXISTENCE OF CONNECTED COMPONENTS OF DIMENSION ONE IN THE BRANCH LOCUS OF MODULI SPACES OF RIEMANN SURFACES
}

\author{
ANTONIO F. COSTA and MILAGROS IZQUIERDO
}

\begin{abstract}
Let $g$ be an integer $\geq 3$ and let $\mathscr{B}_{g}=\left\{X \in \mathscr{M}_{g}: \operatorname{Aut}(X) \neq \mathrm{Id}\right\}$ be the branch locus of $\mathscr{M}_{g}$, where $\mathcal{M}_{g}$ denotes the moduli space of compact Riemann surfaces of genus $g$. The structure of $\mathscr{B}_{g}$ is of substantial interest because $\mathscr{B}_{g}$ corresponds to the singularities of the action of the modular group on the Teichmüller space of surfaces of genus $g$ (see [14]).

Kulkarni ([15], see also [13]) proved the existence of isolated points in the branch loci of the moduli spaces of Riemann surfaces. In this work we study the isolated connected components of dimension 1 in such loci. These isolated components of dimension one appear if the genus is $g=p-1$ with $p$ prime $\geq 11$. We use uniformization by Fuchsian groups and the equisymmetric stratification of the branch loci.
\end{abstract}

\section{Introduction}

In this article we study the topology of moduli spaces of Riemann surfaces, more concretely the existence of dimension one connected components in the singular set of the orbifold structure of the moduli spaces.

Let $g$ be an integer $\geq 3$ and let $\mathscr{B}_{g}=\left\{X \in \mathscr{M}_{g}: \operatorname{Aut}(X) \neq \mathrm{Id}\right\}$, where $\mathcal{M}_{g}$ denotes the moduli space of compact Riemann surfaces of genus $g$. Two closed Riemann surfaces of genus $g$ are called equisymmetric if their automorphism groups determine conjugate finite subgroups of the modular group of genus $g$. An equisymmetric equivalence class will be called equisymmetric stratum or simply stratum. The equisymmetric strata $\mathscr{M}^{G, i}$ are in correspondence with topological equivalence classes of orientation preserving actions of a finite group $G$ on a surface $X, i: G \rightarrow \operatorname{Homeo}(X)$. In this way we have stratifications:

$$
\mathscr{M}_{g}=\bigcup \overline{\mathscr{M}}^{G, i}, \quad \mathscr{B}_{g}=\bigcup_{G \neq\{\mathrm{Id}\}} \overline{\mathscr{M}}^{G, i}
$$

Harvey ([14]) alluded to the existence of the equisymmetric stratification of the moduli space $\mathcal{M}_{g}$. Broughton ([3]) showed that the equisymmetric stratification is indeed a stratification of $\mathscr{M}_{g}$ by irreducible algebraic subvarieties

Received 9 March 2010, in final form 20 July 2011. 
$\overline{\mathcal{M}}^{G, i}$ whose interior, if it is non-empty, is a smooth, connected, locally closed algebraic subvariety of $\mathscr{M}_{g}$, Zariski dense in the stratum.

In this work we show, by means of Fuchsian groups, that $\mathscr{B}_{g}$ contains isolated strata of dimension one if and only if $g=p-1$, with $p$ a prime number, $p \geq 11$. By isolated strata we mean strata that are connected components of $\mathscr{B}_{g}$.

The results in this work have been announced in [2]. Similar results for strata of dimension 1 in the branch locus were found by Urzúa in [20] using different techniques.

Note that $\mathscr{B}_{g}=\bigcup \overline{\mathscr{M}}^{C_{p}, i}$, where $C_{p}$ is a cyclic group of prime order $p$ (see [11]). Recently Bartolini and Izquierdo showed in [1] that all the closed strata in $\mathscr{B}_{g}$ given by actions of $C_{2}$ are connected to the stratum of Riemann surfaces admitting an involution with quotient Riemann surface with two cone points, if $g$ even, and no cone points if $g$ odd. They showed also that any stratum induced by an action of $C_{3}$ is connected to a suitable stratum associated to an action of $C_{2}$.

ACKNOWLEDGEMENT. The first author is partially supported by MTM201123092 and second author by the Swedish Research Council (VR). The authors wish to thank the referee for useful suggestions.

\section{Riemann surfaces and Fuchsian groups}

A Fuchsian group $\Gamma$ is a discrete subgroup of the group of orientation preserving isometries of the hyperbolic unit disc $\mathscr{D}$.

Let $\Gamma$ be a (cocompact) Fuchsian group. The algebraic structure of $\Gamma$ and the orbifold structure of $X_{g}=\mathscr{D} / \Gamma$ are given by the signature of $\Gamma$ :

$$
s(\Gamma)=\left(g ; m_{1}, \ldots, m_{r}\right) .
$$

If $r=0$, then we say that $\Gamma$ is a surface Fuchsian group. The group with the signature (2.1) has a canonical presentation given by generators:

(a) $x_{i}, i=1, \ldots, r$ (elliptic transformations),

(b) $a_{i}, b_{i}, i=1, \ldots, g$ (hyperbolic translations),

and relations:

(1) $x_{i}^{m_{i}}=1, i=1, \ldots, r$,

(2) $x_{1} \ldots x_{r} a_{1} b_{1} a_{1}^{-1} b_{1}^{-1} \ldots a_{g} b_{g} a_{g}^{-1} b_{g}^{-1}=1$.

The hyperbolic area of the orbifold $\mathscr{D} / \Gamma$ coincides with the hyperbolic area of an arbitrary fundamental region of $\Gamma$ and equals:

$$
\mu(\Gamma)=2 \pi\left(2 g-2+\sum_{i=1}^{r}\left(1-\frac{1}{m_{i}}\right)\right),
$$


Given a subgroup $\Gamma^{\prime}$ of index $N$ in a Fuchsian group $\Gamma$, one can calculate the structure of $\Gamma^{\prime}$ by:

THeOrem 1 ([17]). Let $\Gamma$ be a Fuchsian group with signature (2.1) and canonical presentation (2). Then $\Gamma$ contains a subgroup $\Gamma^{\prime}$ of index $N$ with signature

$$
s\left(\Gamma^{\prime}\right)=\left(h ; m_{11}^{\prime}, m_{12}^{\prime}, \ldots, m_{1 s_{1}}^{\prime}, \ldots, m_{r 1}^{\prime}, \ldots, m_{r s_{r}}^{\prime}\right) .
$$

if and only if there exists a transitive permutation representation $\theta: \Gamma \rightarrow \Sigma_{N}$ satisfying the following conditions:

(1) The permutation $\theta\left(x_{i}\right)$ has precisely $s_{i}$ cycles of lengths $m_{i} / m_{i 1}^{\prime}, \ldots$, $m_{i} / m_{i s_{i}}^{\prime}$.

(2) The Riemann-Hurwitz formula

$$
\mu\left(\Gamma^{\prime}\right) / \mu(\Gamma)=N
$$

Let $X$ be a compact Riemann surface of genus $g \geq 2$, then $X$ can be represented as a quotient $X=\mathscr{D} / \Gamma$ of the unit disc $\mathscr{D}$ under the action of a surface Fuchsian group $\Gamma$. A finite group $G$ is a group of automorphisms of $X$ if and only if there exists a Fuchsian group $\Delta$ and an epimorphism $\theta: \Delta \rightarrow G$ with $\operatorname{ker}(\theta)=\Gamma$.

Definition 1. A closed Riemann surface $X$ which can be realized as a $p$-sheeted covering of the Riemann sphere is said to be $p$-gonal, and such a covering will be called a $p$-gonal morphism. When $p=2$, the surface will be called hyperelliptic. Let $\mathscr{M}_{g}^{p} \subset \mathscr{M}_{g}$ denote the locus of $p$-gonal Riemann surfaces of genus $g$.

Trigonal and $p$-gonal Riemann surfaces have been widely studied recently, see [4], [5], [7], [8], [9], [10], [12] and [21].

Let $s$ be a signature of Fuchsian groups and let $\mathscr{G}$ be an abstract group isomorphic to the Fuchsian groups with signature $s$. We denote by $\mathrm{R}(s)$ the set of monomorphisms $r: \mathscr{G} \rightarrow \operatorname{Isom}(\mathscr{D})$ such that $r(\mathscr{G})$ is a Fuchsian group with signature $s$. The set $\mathrm{R}(s)$ has a natural topological structure given by the topology of $\operatorname{Isom}(\mathscr{D})$. Two elements $r_{1}, r_{2} \in \mathrm{R}(s)$ are said to be equivalent, $r_{1} \sim r_{2}$, if there exists $g \in \operatorname{Isom}(\mathscr{D})$ such that for each $\gamma \in \mathscr{G}, r_{1}(\gamma)=$ $g r_{2}(\gamma) g^{-1}$. The space of classes $\mathrm{T}(s)=\mathrm{R}(s) / \sim$ is called the Teichmüller space of Fuchsian groups with signature $s$.

If the signature $s$ is like in (2.1) then the Teichmüller space $\mathrm{T}(s)$ is homeomorphic to a complex ball of dimension $d(\mathrm{~T}(s))=3 g-3+r$ (see [16]). If $\mathscr{G}$ is as above, we say that $\mathscr{G}$ has Teichmüller dimension $d(\mathscr{G})=d(\mathrm{~T}(s))$. Let 
$\Gamma^{\prime} \leq \Gamma$ be Fuchsian groups with signatures $s^{\prime}$ and $s$ respectively. The inclusion mapping $\alpha: \Gamma \rightarrow \Gamma^{\prime}$ induces an embedding $\mathrm{T}(\alpha): \mathrm{T}(s) \rightarrow \mathrm{T}\left(s^{\prime}\right)$ defined by $[r] \mapsto[r \alpha]$ (see [16] and [18]). The modular $\operatorname{group} \operatorname{Mod}(s)$ of $s$ is the quotient $\operatorname{Mod}(s)=\operatorname{Aut}(\mathscr{G}) / \operatorname{Inn}(\mathscr{G})$. The moduli space of Fuchsian groups with signature $s$ is the quotient $\mathscr{M}(s)=\mathrm{T}(s) / \operatorname{Mod}(s)$ endowed with the quotient topology.

The covering

$$
\mathrm{T}((g ;-)) \rightarrow M_{g}=\mathscr{M}((g ;-))=\mathrm{T}((g ;-)) / \operatorname{Mod}((g ;-))=\mathrm{T}_{g} / \operatorname{Mod}_{g}
$$

is a regular branched covering with branch locus: $\mathscr{B}_{g}=\left\{X \in \mathscr{M}_{g}: \operatorname{Aut}(X) \neq\right.$ Id\}, for $g \geq 3$.

Two closed Riemann surfaces $X, \bar{X}$ of genus $g$ are called equisymmetric if their full automorphisms groups determine conjugate subgroups of the modular group of genus $g$.

Let $X$ be a surface, we denote $\operatorname{Homeo}(X)$ the group of orientation preserving autohomeomorphisms of $X$. An (effective and orientable) action of a finite group $G$ on a surface $X$ is a representation $i: G \rightarrow \operatorname{Homeo}(X)$. Two actions $i, i^{\prime}$ of $G$ on a Riemann surface $X$ are (weakly) topologically equivalent if there is a $w \in \operatorname{Aut}(G)$ and an $h \in \operatorname{Homeo}(X)$ such that $i^{\prime}(g)=h(i \circ w(g)) h^{-1}$. The equisymmetric strata are in correspondence with topological equivalence classes of orientation preserving actions of a finite group $G$ on a surface $X$ (see [3]). Let $\mathscr{M}^{G, i}$ denote the stratum of surfaces with automorphisms group in the conjugacy class of the action $i: G \rightarrow \operatorname{Homeo}(X)$ in the modular group and let $\bar{M}^{G, i}$ denote the set of surfaces having an automorphisms group containing the automorphisms group defined by $i$.

Many authors have alluded to the existence of the equisymmetric stratification, we resume such studies in the following result:

Theorem 2 (Theorem 2.1, p. 106, of [3]). Let $M_{g}$ be the moduli space of Riemann surfaces of genus $g, G$ a finite subgroup of the corresponding modular group $\operatorname{Mod}_{g}$. Then:

(1) $\overline{\mathscr{M}}^{G, i}$ is a closed, irreducible algebraic subvariety of $\mathscr{M}_{g}$.

(2) $\mathcal{M}^{G, i}$, if it is non-empty, is a smooth, connected, locally closed algebraic subvariety of $\mathscr{M}_{g}$, Zariski dense in $\overline{\mathscr{M}}^{G, i}$.

There are only finitely many distinct $\mathscr{M}^{G, i}$.

Remark 1. By Lemma 1 in [14] the $\overline{\mathscr{M}}^{G, i}(G \neq\{\mathrm{Id}\})$ are closed subspaces in the quotient topology of $\mathscr{M}_{g}$ given by $\mathrm{T}_{g} / \operatorname{Mod}_{g}$. If $\overline{\mathscr{M}}^{G, i} \cap \overline{\mathscr{M}}^{H, j}=\varnothing$ for all action $(H, j)(H \neq\{\mathrm{Id}\})$ on genus $g$ surfaces, then $\overline{\mathscr{M}}^{G, i}=\mathscr{M}^{G, i}$ is a connected component of $\mathscr{B}_{g}$ and it will be called an isolated stratum. 
By the so called Nielsen realization theorem, each stratum corresponds with a finite subgroup of the modular group represented as the full group of automorphisms of some compact Riemann surface. To decide whether a given finite group can be the full group of automorphism of some compact Riemann surface we will need all pairs of signatures $s(\Gamma)$ and $s\left(\Gamma^{\prime}\right)$ for some Fuchsian groups $\Gamma$ and $\Gamma^{\prime}$ such that $\Gamma^{\prime}<\Gamma$ and $d(\Gamma)=d\left(\Gamma^{\prime}\right)$. The full list of such pairs of signatures was obtained by Singerman in [18]. To find such full automorphisms groups we need to use the list of finite maximal signatures for Fuchsian groups in [18].

Each action of a finite group $G$ on a surface $X$ is determined by an epimorphism $\theta: \Delta \rightarrow G$ from a Fuchsian group $\Delta$ such that $\operatorname{ker}(\theta)=\Gamma$, where $X=\mathscr{D} / \Gamma$ and $\Gamma$ is a surface Fuchsian group. Observe that the topological dimension $\operatorname{dim}\left(\overline{\mathscr{M}}^{G, i_{\theta}}\right)=d(\Delta)$. The condition $\Gamma$ to be a surface Fuchsian group imposes that the order of the image under $\theta$ of an elliptic generator $x_{i}$ of $\Delta$ is the same as the order of $x_{i}$ and $\theta\left(x_{1}\right) \theta\left(x_{2}\right) \ldots \theta\left(x_{r-1}\right)=\theta\left(x_{r}\right)^{-1}$. Two epimorphisms $\theta_{1}, \theta_{2}: \Delta \rightarrow G$ define two topologically equivalent actions of $G$ on $X_{g}$ if and only if there exist automorphisms $\phi: \Delta \rightarrow \Delta, w: G \rightarrow G$ such that $\theta_{2}=w \circ \theta_{1} \circ \phi^{-1}$ (see [19]).

Let $\mathscr{A}$ be the subgroup of $\operatorname{Aut}(\Delta)$ induced by orientation preserving homeomorphisms of the orbifold $\mathscr{D} / \Delta$. Then, two different epimorphisms $\theta_{1}, \theta_{2}$ : $\Delta \rightarrow G$ define the same class of $G$-actions if and only if they lie in the same $\mathscr{A} \times \operatorname{Aut}(G)$-class.

Considering Fuchsian groups $\Delta$ with Teichmüller dimension zero in the discusion above, Kulkarni found the isolated strata of dimension zero in the branch locus $\mathscr{B}_{g}$ (see a different approach in Cornalba [11] and [13]):

THEOREM 3 ([15] and [13]). The number of isolated points in $\mathscr{B}_{g}$ is 1 if $g=2,[(g-2) / 3]$ if $q=2 g+1$ is a prime $>7$, and 0 otherwise.

Note that the strata of dimension zero are automatically isolated. For dimension one closed strata the study is more involved.

\section{Connected components of dimension 1 in the Branch locus in the moduli space of Riemann surfaces}

In this section we will find the possible isolated strata of dimension 1 in $\mathscr{B}_{g}$, for $g \geq 3$. Observe that $\mathscr{B}_{2}$ consists of those surfaces admitting non-trivial automorphisms other than the hyperelliptic involution. It is well known that $\mathscr{B}_{2}$ is connected excepted for the isolated point in $\mathscr{B}_{2}$ given by the curve with equation $w^{2}=z^{5}-1$ (see [10] and [15]). It is also known that $\mathscr{B}_{3}$ is connected. Costa and Izquierdo, and Bartolini and Izquierdo ([1], [6]) have shown that $\mathscr{B}_{4}$ and $\mathscr{B}_{7}$ are connected, while $\mathscr{B}_{5}$ and $\mathscr{B}_{6}$ are connected excepted for one isolated point in each of them (as in Kulkarni's Theorem [15]). 
Let $X \in \mathscr{B}_{g}$, then $\operatorname{Aut}(X)=G \neq\{\operatorname{Id}\}$, so $X \in \mathscr{M}^{G, \bar{i}}$ Since every finite group $G$ contains an element of prime order $p$, where $p$ divides the order of $|G|$, there is a subgroup $C_{p} \leq G$ acting on $X$ by $i$ (the restriction to $C_{p}$ of the action $\bar{i}$ of $G$ ) then $X \in \overline{\mathscr{M}}^{C_{p}, i}$ for some action $i$ of $C_{p}$ on surfaces of genus $g$. Hence $\mathscr{M}^{G, \bar{i}} \subset \overline{\mathscr{M}}^{C_{p}, i}$ and $\mathscr{B}_{g}=\bigcup \overline{\mathscr{M}}^{C_{p, i}}$.

THEOREM 4 (See [11]). The branch locus consists of the union

$$
\mathscr{B}_{g}=\bigcup_{p \text { prime }} \overline{\mathscr{M}}^{C_{p}, i}
$$

where $\bar{M}^{C_{p}, i}$ is the set of Riemann surfaces of genus $g$ with an automorphism group containing $C_{p}$, the cyclic group of order p, acting on surfaces of genus $g$ in a fixed way given by $i$.

REMARK 2. The components in [11] are not connected components, note that in [11] the author studies the inclusions between the strata $\mathscr{M}^{G, i}$ and no the intersections between the $\overline{\mathscr{M}}^{G, i}$.

Using Riemann-Hurwitz formula and Fuchsian groups of Teichmüller dimension one we obtain:

Lemma 1. Let $g \geq 4$. If the branch locus $\mathscr{B}_{g}$ contains isolated strata of dimension 1 then $g=p-1$, with $p$ a prime.

Proof. By Theorem 4 and Remark 14, the possible isolated strata of dimension 1 will be induced by epimorphisms $\theta: \Delta \rightarrow C_{p}, p$ prime, where $s(\Delta)=(0 ; p, p, p, p)$ or $s(\Delta)=(1 ; p)$, and $\operatorname{Ker}(\theta)=\Gamma$ is a surface Fuchsian group of genus $g$. There are no surface epimorphisms $\theta: \Delta \rightarrow C_{p}$, with $s(\Delta)=(1 ; p)$ since a surface epimorphism must satisfy both $o(\theta(x))=p$ and $1=\theta(a) \theta(b) \theta(a)^{-1} \theta(b)^{-1} \theta(x)=\theta(x)$, since $C_{p}$ is Abelian. Using the Riemann-Hurwitz formula $p\left(-2+\frac{4(p-1)}{p}\right)=2(g-1)$ we obtain that $g=p-1$.

Remark 3. The strata of dimension 1 consist of $p$-gonal Riemann surfaces.

From now on let $g$ be such that $g+1=p$ is prime. By Theorem 4, the isolated strata in $\mathscr{B}_{g}$ of dimension one correspond with maximal actions $\left(C_{p}, \theta\right)$ of the cyclic group $C_{p}$. If $\theta: \Delta \rightarrow C_{p}$ is a monodromy epimorphism such that $\mathscr{D} / \operatorname{ker} \theta$ is a surface in an isolated stratum of dimension 1 of $\mathscr{B}_{g}$, then there is no extension $\theta^{\prime}: \Delta^{\prime} \rightarrow G$, of $\theta$ such that $\operatorname{ker} \theta=\operatorname{ker} \theta^{\prime}, G \gtrless C_{p}$ and $\Delta \leq \Delta^{\prime}$. 
The strategy will be to consider the epimorphisms $\theta: \Delta \rightarrow C_{p}, s(\Delta)=$ $(0 ; p, p, p, p)$, such that $\mathscr{D} / \operatorname{ker} \theta$ is a surface and to study the possible extensions to epimorphisms $\theta^{\prime}: \Delta^{\prime} \rightarrow G$ with $\operatorname{ker} \theta=\operatorname{ker} \theta^{\prime}$. In Propositions 1 to 5 we find such finite groups, Fuchsian groups and epimorphisms $\theta^{\prime}: \Delta^{\prime} \rightarrow G$, when existing.

In order to define epimorphisms $\theta: \Delta \rightarrow C_{p}$, with $\Delta$ a group with signature $(0 ; p, p, p, p)$, we separate the epimorphisms in cases according to how many times the same generator of $C_{p}$, and its inverse, will appear as the image of a canonical generator of $\Delta$. Again, as $\theta$ is a surface epimorphism $\theta\left(x_{4}\right)=$ $\left(\theta\left(x_{1}\right) \theta\left(x_{2}\right) \theta\left(x_{3}\right)\right)^{-1}$. By exhaustion, we shall consider five types of actions of $C_{p}$ on a surface of genus $g=p-1$ : Case $1, \theta\left(x_{1}\right)=\theta\left(x_{2}\right)=\theta\left(x_{3}\right)$; Case 2, $\theta\left(x_{1}\right)=\theta\left(x_{2}\right)=\theta\left(x_{3}\right)^{-1}$; Case 3, $\theta\left(x_{2}\right)=\theta\left(x_{1}\right)^{-1}, \theta\left(x_{3}\right)$ distinct from $\theta\left(x_{1}\right), \theta\left(x_{2}\right)$; Case 4, $\theta\left(x_{2}\right)=\theta\left(x_{1}\right)$ and no inverses; and Case 5 all the images distinct and no pair of inverses appear. The five cases of epimorphisms have representatives as follows:

(Type 1) $\theta_{1}: \Delta \rightarrow C_{p}=\left\langle a: a^{p}=1\right\rangle, \theta_{1}\left(x_{1}\right)=a, \theta_{1}\left(x_{2}\right)=a, \theta_{1}\left(x_{3}\right)=a$, $\theta_{1}\left(x_{4}\right)=a^{-3}$.

(Type 2) $\theta_{2}: \Delta \rightarrow C_{p}, \theta_{2}\left(x_{1}\right)=a, \theta_{2}\left(x_{2}\right)=a, \theta_{2}\left(x_{3}\right)=a^{-1}$, $\theta_{2}\left(x_{4}\right)=a^{-1}$.

(Type 3) $\theta_{3 i}: \Delta \rightarrow C_{p}, \theta_{3 i}\left(x_{1}\right)=a, \theta_{3 i}\left(x_{2}\right)=a^{-1}, \theta_{3 i}\left(x_{3}\right)=a^{i}$, $\theta_{3 i}\left(x_{4}\right)=a^{-i}$, with $2 \leq i \leq \frac{p-1}{2}$.

(Type 4) $\theta_{4 i}: \Delta \rightarrow C_{p}, \theta_{4 i}\left(x_{1}\right)=a, \theta_{4 i}\left(x_{2}\right)=a, \theta_{4 i}\left(x_{3}\right)=a^{i}$, $\theta_{4}\left(x_{4}\right)=a^{p-2-i}, 2 \leq i \leq \frac{p-2}{2}$.

(Type 5) $\theta_{5 i j}: \Delta \rightarrow C_{p}, \theta_{5 i j}\left(x_{1}\right)=a, \theta_{5 i j}\left(x_{2}\right)=a^{i}, \theta_{5 i j}\left(x_{3}\right)=a^{j}$, $\theta_{5 i j}\left(x_{4}\right)=a^{p-1-i-j}$, where $2 \leq i \leq \frac{p-1}{2}, j \notin\{1, p-1, i, p-i\}$ and $p-1-i-j \notin\{1, i, j\}$.

Under the action of $\mathscr{A} \times \operatorname{Aut}(G)$, any epimorphism $\theta: \Delta \rightarrow C_{p}, s(\Delta)=$ $(0 ; p, p, p, p)$, such that $\mathscr{D} / \operatorname{ker} \theta$ is a surface, is equivalent to an epimorphism of the described in the above types. Each epimorphism of type 1 and 2 produces one stratum, denoted $\mathscr{M}^{C_{p, 1}}$ and $\mathscr{M}^{C_{p, 2}}$ in $\mathscr{B}_{p-1}$ while actions of types 3,4 and 5 induce several strata $\mathscr{M}^{C_{p, 3 i}}, \mathscr{M}^{C_{p, 4 i}}, \mathscr{M}^{C_{p, s i j}}$ for $p \geq 11$. Actions of type 5 do exist only if $p \geq 11$. Observe that actions of type 1 and 4 coincide for $p=5$.

Proposition 1. The closed stratum $\overline{\mathscr{M}}^{C_{p, 1}}$ has non-empty intersection with the locus $\mathscr{M}_{p-1}^{3}=\bigcup \bar{M}^{C_{3,0, \theta_{j}}}$ of trigonal Riemann surfaces.

Proof. Consider the Riemann surface $X_{p-1}$ induced by the epimorphism $\phi: \Lambda \rightarrow C_{3 p}=\left\langle a, t: a^{p}=t^{3}=[a, t]=1\right\rangle$ defined as $\phi\left(z_{1}\right)=t$, 
$\phi\left(z_{2}\right)=a, \phi\left(z_{3}\right)=t^{2} a^{-1}$, where $s(\Lambda)=(0 ; 3, p, 3 p)$. By Theorem 1 $s\left(\phi^{-1}(\langle a\rangle)\right)=(0 ; p, p, p, p)$ and $s\left(\phi^{-1}(\langle t\rangle)\right)=(0 ; 3, \stackrel{p+1}{*}, 3)$. Moreover the elliptic generators of $\phi^{-1}(\langle a\rangle)$ are mapped to preimages of $a$ (three times) and $t^{2} a^{-1}$ by the inclusion of $C_{p}$ in $C_{3 p}$. So $\phi$ is an extension of $\theta_{1}$ in (Type 1). Therefore the surface $X_{p-1}$ belongs to $\overline{\mathscr{M}}^{C_{p, 1}} \cap \mathscr{M}_{p-1}^{3}$ as required.

Proposition 2. The stratum $\mathscr{M}^{C_{p, 2}}$ in $\mathscr{B}_{p-1}$ is contained in the hyperelliptic locus $\mathscr{M}_{p-1}^{2}=\overline{\mathscr{M}}^{C_{2,0}}$.

Proof. The epimorphism $\theta_{2}: \Delta(0 ; p, p, p, p) \rightarrow C_{p}$ in (Type 2) extends to the epimorphism $\varphi: \bar{\Delta} \rightarrow D_{2 p}=\left\langle b, t: b^{2 p}=t^{2}=(t b)^{2}=1\right\rangle$ defined by $\varphi\left(y_{1}\right)=b^{p}, \varphi\left(y_{2}\right)=t b^{p}, \varphi\left(y_{3}\right)=t b^{2}, \varphi\left(y_{4}\right)=b^{-2}$, with $s(\bar{\Delta})=(0 ; 2,2,2, p)$. Let $c_{1}: D_{2 p} \rightarrow \Sigma_{p}$ be the $\left\langle b^{p}\right\rangle$-coset representation, applying Theorem 1 to $c_{1} \circ \varphi_{1}$ we see that $s\left(\varphi^{-1}\left(\left\langle b^{p}\right\rangle\right)\right)=(0 ; 2,2 p .2)$. In a similar way, using the $\left\langle b^{2}\right\rangle$ and $\langle t\rangle$-cosets representations we see that, $s\left(\varphi^{-1}\left(\left\langle b^{2}\right\rangle\right)\right)=(0 ; p, p, p, p)$ and $s\left(\varphi^{-1}(\langle t\rangle)\right)=\left(\frac{p-1}{2} ; 2,2\right)$. Moreover the four elliptic generators of $\varphi^{-1}\left(\left\langle b^{2}\right\rangle\right)$ are mapped to the preimages of $b^{2}$ by the inclusion of $C_{p}$ in $D_{2 p}$ determined by $\varphi: b^{2}=a, b^{-2}=a^{-1}, b^{2}=a$ and $b^{-2}=a^{-1}$. Then $\varphi$ is an extension of $\theta_{2}$. Therefore the stratum $\mathscr{M}^{C_{p, 2}} \subset$ $\overline{\mathscr{M}}^{C_{2,0}} \cap \overline{\mathscr{M}}^{C_{2, \frac{p-1}{2}}}$. In particular $\mathscr{M}^{C_{p, 2}} \subset \overline{\mathscr{M}}^{C_{2,0}}$ as stated.

Proposition 3. Each stratum $\mathscr{M}^{C_{p, 3 i}}$ is contained in the locus $\overline{\mathscr{M}}^{C_{2, \frac{p-1}{2}}}$ of Riemann surfaces admitting an involution with quotient a Riemann surface of genus $\frac{p-1}{2}$ and two cone points of order 2 .

Proof. The epimorphisms $\theta_{3 i}: \Delta(0 ; p, p, p, p) \rightarrow C_{p}$ in (Type 3 ) extend to epimorphisms $\varphi_{3 i}: \bar{\Lambda} \rightarrow D_{p}=\left\langle a, t: a^{p}=t^{2}=(s a)^{2}=1\right\rangle, \varphi_{3 i}\left(y_{1}\right)=t$, $\varphi_{3 i}\left(y_{2}\right)=t a^{i-1}, \varphi_{3 i}\left(y_{3}\right)=a, \varphi_{3 i}\left(y_{4}\right)=a^{-i}$, with $s(\bar{\Lambda})=(0 ; 2,2, p, p)$. Using the representations $D_{p} \rightarrow \Sigma_{2}=\mathscr{P}\{\langle a\rangle$-cosets $\}$ and $D_{p} \rightarrow \Sigma_{p}=$ $\mathscr{P}\{\langle t\rangle$-cosets $\}$ and Theorem 1 , we see that $s\left(\varphi_{3 i}^{-1}(\langle a\rangle)\right)=(0 ; p, p, p, p)$ and $s\left(\varphi_{3 i}^{-1}(\langle t\rangle)\right)=\left(\frac{p-1}{2} ; 2,2\right)$, where $\mathscr{P}$ denotes the permutation group of finite set. Moreover the elliptic generators of $\varphi_{3 i}^{-1}(\langle b\rangle)$ are mapped for $\varphi_{3 i}$ to $a, a^{-1}$, $a^{i}$ and $a^{-i}$. So $\varphi_{3 i}$ is an extension of $\theta_{3 i}$. Thus the strata $\mathscr{M}^{C_{p, 3 i}} \subset \overline{\mathcal{M}}^{C_{2, \frac{p-1}{2}}}$ as stated.

Proposition 4. The closed strata $\overline{\mathscr{M}}^{C_{p, 4 i}}, 2 \leq i \leq \frac{p-1}{2}$, have non-empty intersection with the locus $\overline{\mathscr{M}}^{C_{2} \frac{p-1}{2}}$ of Riemann surfaces admitting an involution with quotient a Riemann surface of genus $\frac{p-1}{2}$ and two cone points of order 2 .

Proof. First of all, notice that the counter $i$ in epimorphisms $\theta_{4 i}$ in (Type 4) can be chosen to be odd. 
Now, consider Riemann surfaces $Y_{p-1}^{i}, i \equiv 1 \bmod (2)$, induced by epimorphisms $\alpha_{i}: \bar{\Lambda} \rightarrow C_{2 p}=\left\langle b: b^{2 p}=1\right\rangle$ defined as $\alpha_{i}\left(z_{1}\right)=b^{2}$, $\alpha_{i}\left(z_{2}\right)=b^{i}, \alpha_{i}\left(z_{3}\right)=b^{-i-2}$, where $s(\bar{\Lambda})=(0 ; p, 2 p, 2 p)$. By Theorem 1 $s\left(\alpha_{i}^{-1}\left(\left\langle b^{2}\right\rangle\right)\right)=(0 ; p, p, p, p)$ and $s\left(\alpha_{i}^{-1}\left(\left\langle b^{p}\right\rangle\right)\right)=\left(\frac{p-1}{2} ; 2,2\right)$. Moreover the elliptic generators of $\alpha_{i}^{-1}\left(\left\langle b^{2}\right\rangle\right)$ are mapped to the preimages of $b^{2}$ (twice), $b^{i}$ and $b^{-i-2}$ by the inclusion of $C_{p}$ in $C_{2 p}$ determined by $\alpha_{i}: b^{2}=a, b^{2}=a$, $a^{i}=\left(b^{2}\right)^{i}$ and $a^{-i-2}=\left(b^{2}\right)^{i-2}$. So each $\alpha_{i}$ is an extension of $\theta_{4 i}$. Thus the Riemann surfaces $Y_{p-1}^{i}$ belong to $\overline{\mathscr{M}}^{C_{p, 4 i}} \cap \overline{\mathscr{M}}^{C_{2, \frac{p-1}{2}}}$ as required.

We shall show that the strata (Type 5) are isolated in $\mathscr{B}_{p-1}$. Recall that these strata appear if $p \geq 11$.

Proposition 5. Let $p$ be a prime. The strata $\overline{\mathscr{M}}^{C_{p, 5 i j}}$ in (Type 5) are isolated in $\mathscr{B}_{p-1}$.

Proof. If some $\overline{\mathscr{M}}^{C_{p, 5 i j}}$ is not isolated in $\mathscr{B}_{p-1}$ there are two possibilities: either $\overline{\mathscr{M}}^{C_{p, s i j}}$ is contained in a bigger stratum or $\overline{\mathscr{M}}^{C_{p, i j}}$ intersects another stratum, so $\overline{\mathscr{M}}^{C_{p, s i j}}$ contains some surfaces with larger symmetry.

If $\overline{\mathscr{M}}^{C_{p, 5 i j}}$ is contained in a bigger stratum, the class of epimorphisms $\theta_{5 i j}$ : $\Delta \rightarrow C_{p}$ will extend to either epimorphisms $\varphi: \bar{\Delta} \rightarrow C_{2 p}$, with $s(\bar{\Delta})=$ $(0 ; 2,2, p, p), \varphi\left(y_{1}\right)=b^{p}, \varphi\left(y_{2}\right)=b^{p}, \varphi\left(y_{3}\right)=b^{2}, \varphi\left(y_{4}\right)=b^{-2}$; or epimorphisms $\phi: \bar{\Delta} \rightarrow D_{p}, \phi\left(y_{1}\right)=s, \phi\left(y_{2}\right)=s a^{i-1}, \phi\left(y_{3}\right)=a, \phi\left(y_{4}\right)=$ $a^{-i}$. But the restriction of the epimorphisms $\phi$ or $\varphi$ to the subgroup $\Delta=$ $\varphi^{-1}\left(C_{p}\right)$, respectively $\Delta=\phi^{-1}\left(C_{p}\right)$, is an epimorphism of type 2 or 3 .

Secondly, assume that one closed stratum $\overline{\mathscr{M}}^{C_{p, s i j}}$ intersects another stratum $\overline{\mathscr{M}}^{C_{q, \theta_{h}}}$ at a surface $\bar{X}_{p-1}$. By a theorem of González-Díez (Theorem 1 in [12]) $q$ is a prime distinct from $p$. Since $\bar{X}_{p-1} / \operatorname{Aut}\left(\bar{X}_{p-1}\right)$ is an orbifold isomorphic to $\mathscr{D} / \Delta / G$, where $G \leq \operatorname{Aut}(\mathscr{D} / \Delta)$ is the automorphisms group of a platonic solid, then the surface $\bar{X}_{p-1}$ would be uniformized by the kernel of an epimorphism $\phi: \Lambda \rightarrow G$, with $\Lambda$ a triangle Fuchsian group of signature $\left(0 ; m_{1} q^{t_{1}} p^{s_{1}}, m_{2} q^{t_{2}} p^{s_{2}}, m_{3} q^{t_{3}} p^{s_{3}}\right)$ where $|G|=2^{\epsilon} \operatorname{lcm}\left(m_{1} q^{t_{1}} p^{s_{1}}, m_{2} q^{t_{2}} p^{s_{2}}\right.$, $\left.m_{3} q^{t_{3}} p^{s_{3}}\right), m_{1}, m_{2}, m_{3}$ coprime with $q$ and $p ; \epsilon=0,1$. By the RiemannHurwitz formula we have:

$$
|G|\left(-2+\frac{m_{1} q^{t_{1}} p^{s_{1}}-1}{m_{1} q^{t_{1}} p^{s_{1}}}+\frac{m_{2} q^{t_{2}} p^{s_{2}}-1}{m_{2} q^{t_{2}} p^{s_{2}}}+\frac{m_{3} q^{t_{3}} p^{s_{3}}-1}{m_{3} q^{t_{3}} p^{s_{3}}}\right)=2(p-2) .
$$

The only solutions to the Riemann-Hurwitz formula are the following (see also [7], [21]):

(a) $s(\Lambda)=(0 ; p, 2 p, 2 p),|G|=2 p, G=C_{2 p}=\left\langle b \mid b^{2 p}=1\right\rangle$,

(b) $s(\Lambda)=(0 ; 3, p, 3 p),|G|=3 p, G=C_{3 p}=\left\langle b \mid b^{3 p}=1\right\rangle$ 
(c) $s(\Lambda)=(0 ; 2,2 p, 2 p),|G|=4 p$,

(d) $s(\Lambda)=(0 ; 4,4, p),|G|=4 p$,

(e) $s(\Lambda)=(0 ; 2,4,2 p),|G|=8 p$,

(f) $s(\Lambda)=(0 ; 2,3,3 p),|G|=12 p$.

Using Theorem 1 we shall prove that none of the surfaces $\bar{X}_{p-1}$ belongs to a stratum $\overline{\mathscr{M}}^{C_{p, s i j}}$.

(a) The possible epimorphisms $\phi_{i}: \Lambda \rightarrow C_{2 p}$, where $s(\Lambda)=(0 ; p, 2 p$, $2 p)$, are $\phi_{i}\left(z_{1}\right)=b^{2}, \phi_{i}\left(z_{2}\right)=b^{i}, \phi_{i}\left(z_{3}\right)=b^{-(i+2)}$, with $i$ odd, $1 \leq i \leq p-4$ or $i=2 p-1$. The elliptic generators of $\phi_{i}^{-1}\left(\left\langle b^{2}\right\rangle\right)$ have the following images in $C_{p}$ (Propositions 1, 4, and 2 respectively):

$$
\begin{array}{ll}
a=b^{2}, a, a, a^{-3} & \text { if } \quad i=1, \\
a=b^{2}, a, a^{i}, a^{-i-2} & \text { if } \quad 3 \leq i \leq p-4, \quad \text { and } \\
a=b^{2}, a, a^{-1}, a^{-1} & \text { if } \quad i=2 p-1 .
\end{array}
$$

Therefore the surfaces $\bar{X}_{p-1}$ induced by epimorphisms $\phi_{i}$ belong to $\overline{\mathscr{M}}^{C_{p, 1}}$, $\overline{\mathcal{M}}^{C_{p, 4 i}}$ and $\overline{\mathscr{M}}^{C_{p, 2}}$ respectively.

(b) Up to conjugacy class, the only possible epimorphism $\varphi: \Lambda \rightarrow C_{3 p}$, with $s(\Lambda)=(0 ; 3, p, 3 p)$, is the given in Proposition1. Thus the surface $\bar{X}_{p-1}$ induced by epimorphism $\varphi$ belongs to $\overline{\mathscr{M}}^{C_{p, 1}}$.

(c) The epimorphisms in this case are all equivalent to $\psi: \Lambda \rightarrow C_{2 p} \times$ $C_{2}=\left\langle b, t: b^{2 p}=t^{2}=[b, t]=1\right\rangle$, where $s(\Lambda)=(0 ; 2,2 p, 2 p)$, $\psi\left(z_{1}\right)=t, \psi\left(z_{2}\right)=t b, \psi\left(z_{3}\right)=b^{-1}$. Now $s\left(\psi^{-1}\left(\left\langle b^{2}\right\rangle\right)\right)=(0 ; p, p, p, p)$, where the elliptic generators of $\psi^{-1}\left(\left\langle b^{2}\right\rangle\right)$ have images (Proposition 2): $a=$ $b^{2}, a, a^{-1}, a^{-1}$. Therefore the surface $\bar{X}_{p-1}$ induced by an epimorphism $\psi$ belongs to $\overline{\mathscr{M}}^{C_{p, 2}}$.

(d) The epimorphisms in this case are equivalent to two possible types. The first type is represented by $\mu: \Lambda(0 ; 4,4, p) \rightarrow C_{p} \rtimes_{2} C_{4}=\langle a, t:$ $\left.a^{p}=t^{4}=t^{3} a t a=1\right\rangle$ where $\mu\left(z_{1}\right)=t, \mu\left(z_{2}\right)=t a^{i}, \mu\left(z_{3}\right)=a^{-i}$. Hence $s\left(\mu^{-1}(\langle a\rangle)\right)=(0 ; p, p, p, p)$, where the elliptic generators of $\mu^{-1}(\langle a\rangle)$ have images (see Proposition 3): $a^{i}, a^{i}, a^{-i}, a^{-i}$. Therefore the surface $\bar{X}_{p-1}$ induced by an epimorphism $\mu$ belongs to $\bar{M}^{C_{p, 2}}$.

If $p \equiv 1 \bmod (4)$, then there is the second type of epimorphisms, in this case represented by $\bar{\mu}: \Lambda \rightarrow C_{p} \rtimes_{4} C_{4}=\langle a, t| a^{p}=t^{4}=t^{3} a t a^{-x}=1, x^{4} \equiv$ $1 \bmod (p)\rangle$, with $s(\Lambda)=(0 ; 4,4, p)$, and defined by $\bar{\mu}:\left(z_{1}\right)=t, \bar{\mu}\left(z_{2}\right)=$ $t a^{i}, \bar{\mu}\left(z_{3}\right)=a^{-i}$. Thus $s\left(\bar{\mu}^{-1}(\langle a\rangle)\right)=(0 ; p, p, p, p)$, where the elliptic generators of $\bar{\mu}^{-1}(\langle a\rangle)$ have images (see Proposition 3): $a^{i}, a^{-i}, a^{i x}, a^{-i x}$. Therefore the surface $\bar{X}_{p-1}$ induced by an epimorphism $\bar{\mu}$ belongs to $\overline{\mathscr{M}}^{C_{p, 3 i}}$. 
Notice that epimorphisms $\psi$ in case (c) and $\mu$ are extensions of epimorphisms $\phi_{2 p-1}$ in part (a).

(e) In this case the epimorphisms are $\alpha_{i}: \Lambda \rightarrow C_{p} \rtimes_{2} D_{4}=\langle a, r, t|$ $\left.a^{p}=r^{2}=t^{4}=(r t)^{2}=[r, a]=t^{3} a t a=1\right\rangle, s(\Lambda)=(0 ; 2,4,2 p)$, defined by $\alpha_{i}\left(z_{1}\right)=r t, \alpha_{i}\left(z_{2}\right)=t^{3} a^{i}, \alpha_{i}\left(z_{3}\right)=r a^{-i}$. Then $s\left(\alpha_{i}^{-1}(\langle a\rangle)\right)=$ $(0 ; p, p, p, p)$, where the elliptic generators of $\alpha_{i}^{-1}(\langle a\rangle)$ have images (see Propositions 2 and 3): $a^{i}, a^{i}, a^{-i}, a^{-i}$. Therefore the surfaces $\bar{X}_{p-1}$ induced by epimorphisms $\alpha_{i}$ belong to $\overline{\mathscr{M}}^{C_{p, 2}}$.

Notice that epimorphisms $\alpha_{i}$ are extensions of epimorphisms $\psi$ and $\mu$ in cases (c) and (d).

(f) Since $((0 ; 3, p, 3 p),(0 ; 2,3,3 p))$ is a pair of non-maximal signatures ([18]), a possible epimorphism $\beta: \Lambda(0 ; 2,3,3 p) \rightarrow G$ with $|G|=12 p$ would be an extension of the epimorphism $\varphi$ in case (b).

However, one can show that $G=C_{p} \rtimes A_{4}$. Since $C_{p} \rtimes A_{4}$ is not generated by elements of order two and three then such an epimorphism $\beta$ does not exist.

As a consequence of Theorem 4, Lemma 1 and Propositions 1-5 we have:

THEOREM 5. The branch locus $\mathscr{B}_{g}$ of the moduli space of Riemann surfaces of genus g contains isolated connected components of (complex) dimension 1 if and only if $g=p-1$, with $p$ a prime $\geq 11$. Furthermore each connected component of dimension 1 consists of Riemann surfaces with an automorphism of order $p$ with four fixed points and rotation angles in the fixed points $\frac{2 \pi}{p}$, $\frac{2 \pi i}{p}, \frac{2 \pi j}{p}, \frac{2 \pi k}{p}$, with $1<i<j<k<p-1,\{j, k\} \neq\{i, p-i\}, i+j+k=$ $-1 \bmod p$.

ReMARK 4.

(1) As a consequence of Propositions 2, 4 and 5 we obtain that the closed subvariety $\overline{\mathscr{M}}^{2, \frac{p-1}{2}}$ intersects all the closed subvarieties of dimension 1 in $\mathscr{B}_{p-1}, p$ a prime $\leq 3$, except the ones given in Theorem 6.

(2) For $p=3,5,7$ there are no isolated strata of dimension 1 in $\mathscr{B}_{p-1}$ (see also [1] and [6]). The first genus where $\mathscr{B}_{g}$ contains isolated strata of dimension 1 is $g=10$. There is one such stratum in $\mathscr{B}_{10}$.

(3) In case $p=5$, the Riemann surface $\bar{X}_{4}$ induce by $\bar{\mu}: \Lambda(0 ; 4,4,5) \rightarrow$ $C_{5} \rtimes_{4} C_{4}$ is Bring's curve: the curve in $\mathscr{M}_{4}$ with $\operatorname{Aut}\left(\bar{X}_{4}\right)=\Sigma_{5}$.

(5) The first genus where $\mathscr{B}_{g}$ contains isolated points and isolated strata of dimension 1 is $g=18$. 


\section{REFERENCES}

1. Bartolini, G., and Izquierdo, M., On the connectedness of the branch locus of the moduli space of Riemann surfaces of low genus, Proc. Amer. Math. Soc. 140 (2012), 35-45.

2. Bartolini, G., Costa, A. F., Izquierdo, M., and Porto, A. M., On the connectedness of the branch locus of the moduli space of Riemann surfaces, Rev. R. Acad. Cienc. Exactas Fís. Nat. (A) RACSAM 104 (2010), 81-86.

3. Broughton, S. A., The equisymmetric stratification of the moduli space and the Krull dimension of mapping class groups, Topology Appl. 37 (1990), 101-113.

4. Bujalance, E., Cirre, J., and Gromadzki, G., Groups of automorphisms of cyclic trigonal Riemann surfaces, J. Algebra 322 (2009), 1086-1103.

5. Broughton, S. A., Wootton, A., Finite abelian subgroups of the mapping class group, Algebr. Geom. Topol. 7 (2007), 1651-1697.

6. Costa, A. F., and Izquierdo, M., On the connectedness of the branch locus of the moduli space of Riemann surfaces of genus 4, Glasg. Math. J. 52 (2010), 401-408.

7. Costa, A. F., and Izquierdo, M., Symmetries of real cyclic p-gonal Riemann surfaces, Pacific J. Math. 213 (2004), 231-243.

8. Costa, A. F., and Izquierdo, M., On real trigonal Riemann surfaces, Math. Scand. 98 (2006), $53-68$.

9. Costa, A. F., and Izquierdo, M., Maximal order of automorphisms of trigonal Riemann surfaces, J. Algebra 323 (2010), 27-31.

10. Costa, A. F., Izquierdo, M., and Ying, D., On Riemann surfaces with non-unique cyclic trigonal morphisms, Manuscripta Math. 118 (2005), 443-453.

11. Cornalba, M., On the locus of curves with automorphisms, Ann. Mat. Pura Appl. (4) 149 (1987), 135-151 and (Erratum) Ann. Mat. Pura Appl. (4) 187 (2008), 185-186. (Revised version incorporating the changes described in the Erratum can be find in the author's website).

12. González-Díez, G., On prime Galois coverings of the Riemann sphere, Ann. Mat. Pura Appl. 168 (1995), 1-15.

13. Gómez González, E., Irreductible components and isolated points in the branch locus of the moduli space of smooth curves, Bol. Soc. Mat. Mexicana (3) 2 (1996), 115-128.

14. Harvey, W., On branch loci in Teichmüller space, Trans. Amer. Math. Soc. 153 (1971), 387399.

15. Kulkarni, R. S., Isolated points in the branch locus of the moduli space of compact Riemann surfaces, Ann. Acad. Sci. Fen. (A I Math) 16 (1991), 71-81.

16. Nag, S., The Complex Analytic Theory of Teichmüller Spaces, Wiley, New York 1988.

17. Singerman, D., Subgroups of Fuchsian groups and finite permutation groups, Bull. London Math. Soc. 2 (1970), 319-323

18. Singerman, D., Finitely maximal Fuchsian groups, J. London Math. Soc. (2) 6 (1972), 29-38.

19. Smith, P. A., Abelian actions on 2-manifolds, Michigan Math. J. 14 (1967), 257-275.

20. Urzúa, G., Riemann surfaces of genus $g$ with an automorphism of order p prime and $p>g$, Manuscripta Math. 121 (2006), 169-189.

21. Wootton, A., The full automorphism group of a cyclic p-gonal surface, J. Algebra 312 (2007), 377-396.

DEPARTAMENTO DE MATEMÁTICAS FUNDAMENTALES

FACULTAD DE CIENCIAS

UNED

28040 MADRID

SPAIN

E-mail: acosta@mat.uned.es
MATEMATISKA INSTITUTIONEN LINKÖPINGS UNIVERSITET 58183 LINKÖPING

SWEDEN

E-mail: miizq@mai.liu.se 